Communication

\title{
A New One-Pot Synthesis of Quinoline-2- carboxylates under Heterogeneous Conditions
}

\author{
Serena Gabrielli, Alessandra Giardinieri, Susanna Sampaolesi, Roberto Ballini \\ and Alessandro Palmieri *
}

Green Chemistry Group, Chemistry Division, School of Science and Technology, University of Camerino, via S. Agostino 1, 62032 Camerino, Italy; serena.gabrielli@unicam.it (S.G.);

alessand.giardinieri@studenti.unicam.it (A.G.); susanna.sampaolesi@unicam.it (S.S.); roberto.ballini@unicam.it (R.B.)

* Correspondence: alessandro.palmieri@unicam.it; Tel.: +39-0737-402-262

Academic Editor: Derek J. McPhee

Received: 24 May 2016; Accepted: 8 June 2016; Published: 15 June 2016

\begin{abstract}
Quinoline-2-carboxylates are an important subclass of quinoline derivatives largely present in a variety of biologically active molecules, as well as useful ligands in metal-catalyzed reactions. Herein, we present a new one-pot protocol for synthesizing this class of derivatives starting from $\beta$-nitroacrylates and 2-aminobenzaldehydes. In order to optimize the protocol, we investigated several reaction conditions, obtaining the best results using the 2-tert-Butylimino-2-diethylamino1,3-dimethylperhydro-1,3,2-diazaphosphorine (BEMP) as solid base, in acetonitrile. Finally, we demonstrated the generality of our approach over several substrates which led to synthesize a plethora of functionalized quinolines-2-carboxylate derivatives in good overall yields.
\end{abstract}

Keywords: quinolines; $\beta$-nitroacrylates; one-pot processes; solid supported reagents; heterocycles

\section{Introduction}

Quinoline constitutes one of the most important nitrogen-containing heterocyclic compounds possessing several biological activities such as anti-bacterial, anti-fungal, anti-malarial, anti-convulsant, anti-inflammatory, anthelmintic, cardiotonic and analgesic [1]. In addition, the quinolinic nucleus is largely occurring in natural compounds and deeply investigated as building blocks for synthesizing highly functionalized materials [2,3]. In particular, quinoline-2-carboxylates play a predominant role as precursor of biologically active molecules [4-8] and as useful ligands in metal-catalyzed reactions $[9,10]$. Due to their importance, over the years, a variety of synthetic methodologies have been reported in the literature, which can be classified (Scheme 1) in: (i) oxidative processes, starting from the corresponding 2-methyl or 2-carbonyl quinolines [11,12]; (ii) hydrolytic reactions of cyano precursors [13]; (iii) metal-catalyzed cyclization, starting from $N$-aryl glycine derivatives or iminoethyl glyoxylates [14,15]; (iv) one-pot reduction-cyclization processes of 2-acylnitroarenes with $\alpha$-oxoesters [16]; (v) carboxylation reactions of 2-chloro quinoline derivatives [17]; and (vi) the historical Doebner-Von Miller protocol [18].

Nevertheless, several of the reported procedures present some limitations such as restricted applicability (only few examples were reported) [11-13,18], low overall yields [16] and harsh reaction conditions [17]. Hence, new simple and general protocols for synthesizing these derivatives would surely be welcome. In this context, following our studies on the chemistry of $\beta$-nitroacrylates as valuable precursors of heterocycle systems [19-27], we propose their application as strategic starting materials, in combination with 2-aminobenzaldheydes, for synthesizing quinoline-2-carboxylates. The idea was to develop a new one-pot process involving four different reactions: (i) an $a z a$-Michael addition between the 2-aminobenzaldheydes $\mathbf{1}$ and $\beta$-nitroacrylates $\mathbf{2}$; followed by (ii) an 
intramolecular Henry reaction to give the benzopiperidines 3; (iii) elimination of water; and (iv) nitrous acid elimination to provide the aromatization of the piperidine core, and thus the formation of title targets 4 (Scheme 2).

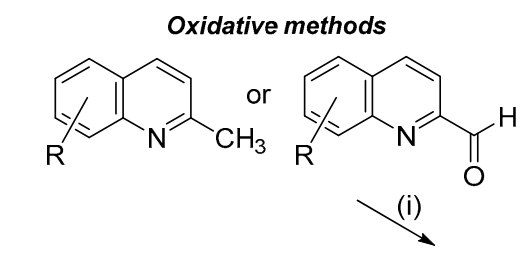

Hydrolytic methods<smiles></smiles>

Carboxylation methods

$$
\text { Quinoline-2-carboxylates (iv) (ii) }
$$

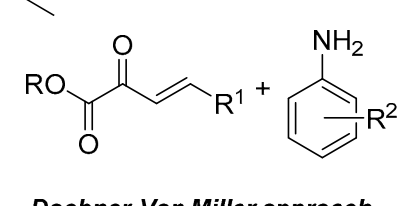

Scheme 1. Main synthetic approaches for synthesizing quinoline-2-carboxylate derivatives.

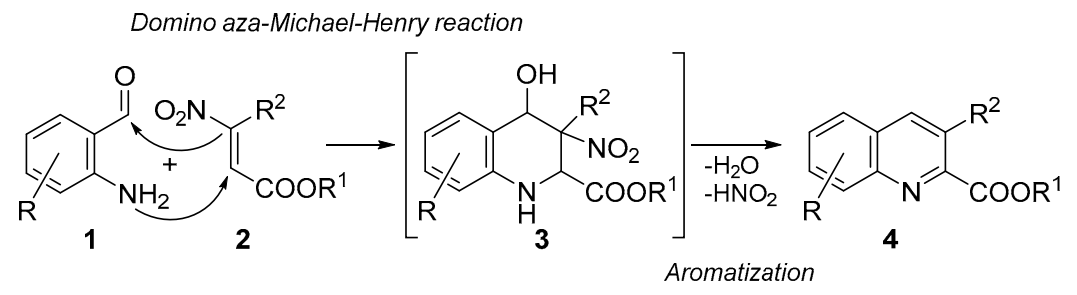

Scheme 2. Synthetic approach for synthesizing quinoline-2-carboxylate derivatives 4 .

\section{Results and Discussion}

In order to study the reaction, we first synthesized the starting materials (Scheme 3). 2-Aminobenzaldheydes were prepared from the corresponding alcohols or nitro precursors [28,29], while $\beta$-nitroacrylates were synthesized by the Henry reaction-elimination process, starting from nitroalkanes and alkyl glyoxalates [30,31].
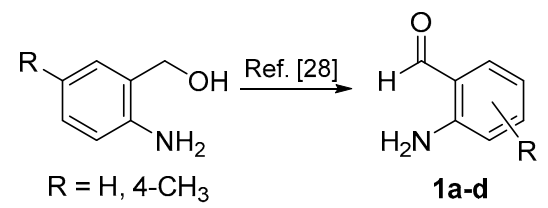<smiles>[R]c1cccc(C(=O)C=CC(C)C)c1[N+](=O)[O-]</smiles>

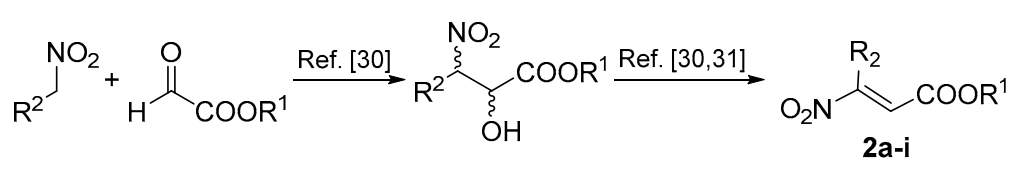

Scheme 3. Synthesis of starting materials $\mathbf{1}$ and $\mathbf{2}$.

Once the starting materials were prepared, we switched our attention to optimize the process. Thus, first we studied the "aza-Michael-Henry domino process" between 2-aminobenzaldheyde 1a and ethyl-3-nitropent-2-enoate $\mathbf{2 a}$ (Table 1). Based on our previous experiences, we started testing the 
reaction under promoter-free and solvent-free conditions and, after a series of trials, the best result was obtained using a slight excess of $2 \mathrm{a}\left(1.1\right.$ equivalent) at $70{ }^{\circ} \mathrm{C}$ (3aa, $66 \%$, entry 7 , the presence of solvents or bases makes the reaction unproductive entry 9-12).

Table 1. Optimization studies concerning the "aza-Michael-Henry domino process".

\begin{tabular}{|c|c|c|c|c|c|c|}
\hline Entry & 1a (mmol) & 2a (mmol) & Solvent & Temp. $\left({ }^{\circ} \mathrm{C}\right)$ & Time (h) & Yield (\%) $^{1}$ \\
\hline Entry 1 & 1 & 1 & - & r.t. & 24 & Traces \\
\hline Entry 2 & 1 & 1 & - & 50 & 24 & 44 \\
\hline Entry 3 & 1 & 1 & - & 60 & 24 & 51 \\
\hline Entry 4 & 1 & 1 & - & 70 & 18 & 61 \\
\hline Entry 5 & 1 & 1 & - & 80 & 18 & 52 \\
\hline Entry 6 & 1.1 & 1 & - & 70 & 18 & 59 \\
\hline Entry 7 & 1 & 1.1 & - & 70 & 18 & 66 \\
\hline Entry 8 & 1 & 1.2 & - & 70 & 18 & 64 \\
\hline Entry 9 & 1 & 1.1 & $\mathrm{MeCN}$ & 70 & 18 & 11 \\
\hline Entry 10 & 1 & 1.1 & EtOAc & 70 & 18 & 9 \\
\hline Entry 11 & 1 & 1.1 & - & 70 & 18 & $21^{2}$ \\
\hline Entry 12 & 1 & 1.1 & - & 70 & 18 & $8^{3}$ \\
\hline
\end{tabular}

Successively, we studied the conversion of 3aa into 4aa (Table 2) and, after deep screening in terms of bases, solvents and temperature, we found the best yield of $4 \mathbf{a a}(86 \%$, entry 8$)$ at $50{ }^{\circ} \mathrm{C}$, in acetonitrile using 1.25 equivalents of BEMP on polymer [32].

Table 2. Optimization studies concerning aromatization step.

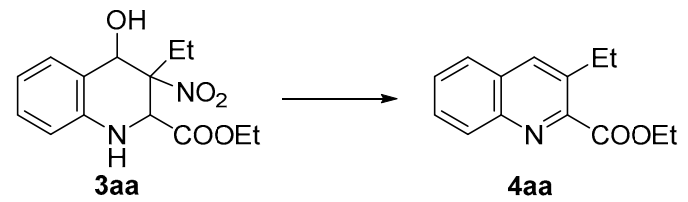

\begin{tabular}{cccccc}
\hline Entry & Base (eq.) & Solvent & Temp. $\left({ }^{\circ} \mathbf{C}\right)$ & Time (h) & Yield (\%) ${ }^{\mathbf{1}}$ \\
\hline Entry 1 & TMG (1) & $\mathrm{MeCN}$ & 50 & 24 & 47 \\
Entry 2 & DBU (1) & $\mathrm{MeCN}$ & 50 & 24 & 36 \\
Entry 3 & Amberlyst A21 (1) & $\mathrm{MeCN}$ & 50 & 24 & Traces \\
Entry 4 & TBD on polymer (1) & $\mathrm{MeCN}$ & 50 & 24 & 62 \\
Entry 5 & Carbonate on polymer (1) & $\mathrm{MeCN}$ & 50 & 24 & 57 \\
Entry 6 & BEMP on polymer (1) & $\mathrm{MeCN}$ & 50 & 24 & 79 \\
Entry 7 & BEMP on polymer (0.8) & $\mathrm{MeCN}$ & 50 & 24 & 67 \\
Entry 8 & BEMP on polymer (1.25) & $\mathrm{MeCN}$ & 50 & 24 & 86 \\
Entry 9 & BEMP on polymer (1.5) & $\mathrm{MeCN}$ & 50 & 24 & 82 \\
Entry 10 & BEMP on polymer (1.25) & $\mathrm{MeCN}$ & 40 & 24 & 69 \\
Entry 11 & BEMP on polymer (1.25) & $\mathrm{MeCN}$ & 60 & 24 & 85 \\
Entry 12 & BEMP on polymer (1.25) & EtOAc & 50 & 24 & 71 \\
Entry 13 & BEMP on polymer (1.25) & 2-MeTHF & 50 & 24 & 47 \\
Entry 14 & BEMP on polymer (1.25) & Toluene & 50 & 24 & 39 \\
\hline
\end{tabular}

${ }^{1}$ Yield of pure isolated product.

At that point, in order to achieve a one-pot process, we combined the two steps obtaining the quinoline 4 aa in $58 \%$ of overall yield (Scheme 4 ). 


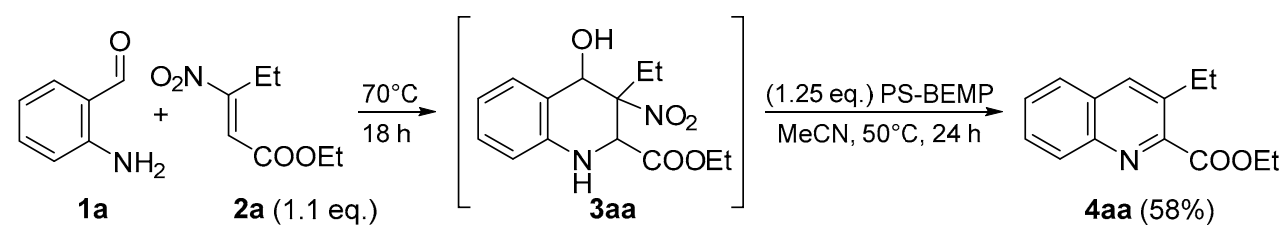

Scheme 4. One-pot process.

Finally, with the aim to demonstrate the generality of our method, we tested our reaction conditions on a wide range of 2 -aminobenzaldheydes $\mathbf{1}$ and $\beta$-nitroacrylates $\mathbf{2}$. In all cases, the products were obtained in moderate to good overall yields $(37 \%-64 \%)$, even in the presence of a variety of functionalities (Scheme 5).
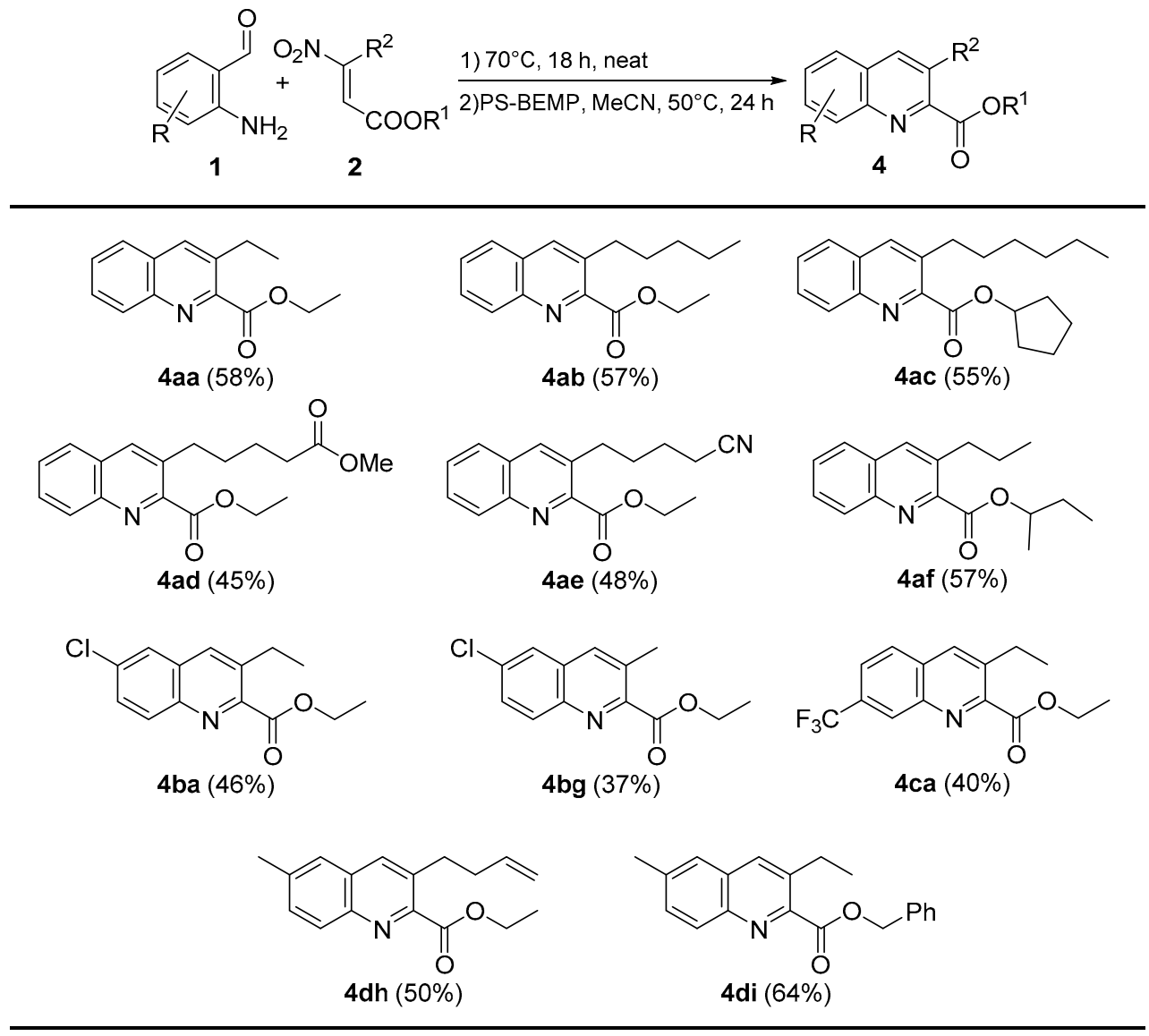

Scheme 5. One-pot reaction products.

\section{Conclusions}

In conclusion, we developed a new general and valuable one-pot procedure for synthesizing an important class of quinolines such as quinoline-2-carboxylates. In our approach, it was possible to prepare title compounds in good overall yields, introducing different substituent in 3-position as well as in the benzene ring. Furthermore, the mildness of our reaction conditions allowed preserving several functionalities such as ester, cyano, chlorine, fluorine and carbon-carbon double bond. In addition, the use of supported BEMP enabled us to minimize the use of materials, avoiding any complex aqueous work-up, with evident advantages from a sustainable point of view. Finally, we still demonstrated the usefulness of $\beta$-nitroacrylates as a valuable precursor of heterocyclic systems. 


\section{Experimental Section}

\subsection{General Section}

${ }^{1} \mathrm{H}-\mathrm{NMR}$ were recorded at $400 \mathrm{MHz}$ on a VarianMercury Plus $400 .{ }^{13} \mathrm{C}-\mathrm{NMR}$ were recorded at $100 \mathrm{MHz}$. IR spectra were recorded with a PerkineElmer Paragon 500 FT-IR. Mass spectra were performed on a GC/MS system by means of the EI technique $(70 \mathrm{eV})$. Microanalyses were performed with a CHNS-O analyzer Model EA 1108 from Fisons Instruments.

\subsection{Chemistry Section}

General Procedure for the Preparation of Compounds 4

A mixture of 2-aminobenzaldheydes $1(1 \mathrm{mmol})$ and $\beta$-nitroacrylates $2(1.1 \mathrm{mmol})$ was stirred, under solvent-free conditions, at $70{ }^{\circ} \mathrm{C}$ for $18 \mathrm{~h}$. Then, after that the temperature was diminished at $50{ }^{\circ} \mathrm{C}$, acetonitrile $(10 \mathrm{~mL})$ and PS-BEMP $(1.25 \mathrm{mmol}, 0.570 \mathrm{mg})$ were added, and the resulting solution was stirred at $50{ }^{\circ} \mathrm{C}$ for further $24 \mathrm{~h}$. Finally, the resin was filtered off washing with fresh EtOAC $(10 \mathrm{~mL})$ and the crude products 4 , obtained after removal of the solvent at reduced pressure, were purified by flash chromatography column (hexane-ethyl acetate 9:1).

Compound 4aa. Pale yellow oil. IR ( $\mathrm{cm}^{-1}$, neat): 1063, 1160, 1458, 1619, 1727, 2959. ${ }^{1} \mathrm{H}-\mathrm{NMR}\left(\mathrm{CDCl}_{3}\right.$, $400 \mathrm{MHz}) \delta: 1.34(\mathrm{t}, 3 \mathrm{H}, J=7.7 \mathrm{~Hz}), 1.46(\mathrm{t}, 3 \mathrm{H}, J=7.3 \mathrm{~Hz}), 3.01(\mathrm{q}, 2 \mathrm{H}, J=7.7 \mathrm{~Hz}), 4.53(\mathrm{q}, 2 \mathrm{H}$, $J=7.3 \mathrm{~Hz}), 7.53-7.60(\mathrm{~m}, 1 \mathrm{H}), 7.65-7.72(\mathrm{~m}, 1 \mathrm{H}), 7.78(\mathrm{~d}, 1 \mathrm{H}, J=8.1 \mathrm{~Hz}), 8.04(\mathrm{~s}, 1 \mathrm{H}), 8.16(\mathrm{~d}, 1 \mathrm{H}$, $J=8.5 \mathrm{~Hz}) .{ }^{13} \mathrm{C}-\mathrm{NMR}\left(\mathrm{CDCl}_{3}, 100 \mathrm{MHz}\right) \delta: 14.5,15.4,25.9,62.2,127.2,128.2,129.1,129.5,130.0,135.6$, 136.4, 146.0, 150.7, 167.1. GC-MS (70 eV): $m / z: 229$ ([M+1, 29), 200 (83), 182 (14), 157 (39), 156 (99), 155 (63), 154 (100), 128 (34). Anal. Calcd. for $\mathrm{C}_{14} \mathrm{H}_{15} \mathrm{NO}_{2}$ (229.28): C, 73.34; $\mathrm{H}, 6.59 ; \mathrm{N}, 6.11$. Found: $\mathrm{C}$, 73.39; H, 6.62; N, 6.08 .

Compound 4ab. Pale yellow oil. IR ( $\mathrm{cm}^{-1}$, neat): 1073, 1171, 1459, 1610, 1728, 2952. ${ }^{1} \mathrm{H}-\mathrm{NMR}\left(\mathrm{CDCl}_{3}\right.$, $400 \mathrm{MHz}) \delta: 0.9(\mathrm{t}, 3 \mathrm{H}, J=7.3 \mathrm{~Hz}), 1.34-1.41(\mathrm{~m}, 4 \mathrm{H}), 1.47(\mathrm{t}, 3 \mathrm{H}, J=7.3 \mathrm{~Hz}), 1.65-1.74(\mathrm{~m}, 2 \mathrm{H}), 2.96-2.99$ $(\mathrm{m}, 2 \mathrm{H}), 4.53(\mathrm{q}, 2 \mathrm{H}, J=\mathrm{Hz} 7.3), 7.55-7.60(\mathrm{~m}, 1 \mathrm{H}), 7.67-7.72(\mathrm{~m}, 1 \mathrm{H}), 7.79(\mathrm{~d}, 1 \mathrm{H}, J=8.1 \mathrm{~Hz}), 8.04(\mathrm{~s}$, $1 \mathrm{H}), 8.175(\mathrm{~d}, 1 \mathrm{H} \mathrm{J}=8.5 \mathrm{~Hz}) .{ }^{13} \mathrm{C}-\mathrm{NMR}\left(\mathrm{CDCl}_{3}, 100 \mathrm{MHz}\right) \delta: 14.1,14.4,22.6,30.1,31.8,32.7,62.1,127.1$, 128.1, 129.0, 129.4, 130, 134.3, 137.1, 146.0, 151.0, 167.1. GC-MS (70 eV): $m / z: 271\left(\left[\mathrm{M}^{+}\right], 25\right), 245(68)$, 228 (72), 224 (44), 199 (14), 198 (96), 196 (26), 182 (35), 168 (38), 166 (29), 156 (22), 155 (20), 154 (100), 143 (72), 142 (43), 141 (15), 140 (19), 132 (14), 115 (38). Anal. Calcd. for $\mathrm{C}_{17} \mathrm{H}_{21} \mathrm{NO}_{2}$ (271.36): C, 75.25; $\mathrm{H}$, 7.80; N, 5.16. Found: C, 75.29; H, 7.77; N, 5.13.

Compound 4ac. Pale yellow oil. IR ( $\mathrm{cm}^{-1}$, neat): 1069, 1167, 1455, 1615, 1722, 2955. ${ }^{1} \mathrm{H}-\mathrm{NMR}\left(\mathrm{CDCl}_{3}\right.$, $400 \mathrm{MHz}) \delta:$ 0.84-0.92 (m, 3H), 1.23-1.44 (m, 6H), 1.59-1.73 (m, 4H), 1.74-1.86 (m, 2H), 1.89-2.11 (m, $4 \mathrm{H}), 2.88-2.94(\mathrm{~m}, 2 \mathrm{H}), 5.51-5.58(\mathrm{~m}, 1 \mathrm{H}), 7.53-7.58(\mathrm{~m}, 1 \mathrm{H}), 7.71-7.75(\mathrm{~m}, 1 \mathrm{H}), 7.77(\mathrm{~d}, 1 \mathrm{H}, J=8.1 \mathrm{~Hz})$, $8.02(\mathrm{~s}, 1 \mathrm{H}), 8.17(\mathrm{~d}, 1 \mathrm{H}, J=8.5 \mathrm{~Hz}) .{ }^{13} \mathrm{C}-\mathrm{NMR}\left(\mathrm{CDCl}_{3}, 100 \mathrm{MHz}\right) \delta: 14.3,22.8,24.1,29.5,31.4,31.9,32.8$, $32.9,79.4,127.2,128.1,128.9,129.6,129.8,133.7,137.2,145.8,151.5,167.2$. GC-MS $(70 \mathrm{eV}): \mathrm{m} / z: 325$ ([M+ ], 4), 257(14), 256 (73), 238 (14), 213 (18), 212 (100), 200 (20), 182 (17), 168 (19), 157 (16), 155 (26), 143 (55), 142 (61), 115(20). Anal. Calcd. for $\mathrm{C}_{21} \mathrm{H}_{27} \mathrm{NO}_{2}$ (325.45): C, 77.50; $\mathrm{H}, 8.36 ; \mathrm{N}, 4.30$. Found: C, 77.55; $\mathrm{H}, 8.39 ; \mathrm{N}, 4.27$.

Compound 4ad. Pale yellow oil. IR ( $\mathrm{cm}^{-1}$, neat): 1074, 1179, 1436, 1615, 1730, 2949. ${ }^{1} \mathrm{H}-\mathrm{NMR}\left(\mathrm{CDCl}_{3}\right.$, $400 \mathrm{MHz}) \delta: 1.46(\mathrm{t}, 3 \mathrm{H}, J=7.3 \mathrm{~Hz}), 1.70-1.76(\mathrm{~m}, 4 \mathrm{H}), 2.31-2.39(\mathrm{~m}, 2 \mathrm{H}), 2.91-3.02(\mathrm{~m}, 2 \mathrm{H}), 3.65(\mathrm{~s}, 3 \mathrm{H})$, $4.50(\mathrm{q}, 2 \mathrm{H}, J=7.3 \mathrm{~Hz}), 7.57(\mathrm{t}, 1 \mathrm{H}, J=7.3 \mathrm{~Hz}), 7.68(\mathrm{t}, 1 \mathrm{H}, J=7.3 \mathrm{~Hz}), 7.77(\mathrm{~d}, 1 \mathrm{H}, J=8.1 \mathrm{~Hz}), 8.02(\mathrm{~s}$, $1 \mathrm{H}), 8.15(\mathrm{~d}, 1 \mathrm{H}, J=8.5 \mathrm{~Hz}) .{ }^{13} \mathrm{C}-\mathrm{NMR}\left(\mathrm{CDCl}_{3}, 100 \mathrm{MHz}\right) \delta: 14.5,24.9,30.8,32.6,34.0,51.8,62.3,127.2$, 128.3, 129.0, 129.7, 130.0, 133.8, 137.4, 146.0, 150.5, 167.0, 174.1. GC-MS (70 eV): $\mathrm{m} / z: 315\left(\left[\mathrm{M}^{+}\right], 30\right), 286$ (68), 284 (29), 270 (18), 242 (28), 236 (21), 228 (81), 182 (61), 180 (29), 170 (14), 168 (42), 167 (44), 156 (14), 155(22), 154 (100), 143 (52), 142 (22), 127 (14), 115 (27). Anal. Calcd. for $\mathrm{C}_{18} \mathrm{H}_{21} \mathrm{NO}_{4}$ (315.37): C, 68.55; H, 6.71; N, 4.44. Found: C, 68.59; H, 6.68; N, 4.47. 
Compound 4ae. Pale yellow oil. IR ( $\mathrm{cm}^{-1}$, neat): 1027, 1236, 1456, 1659, 1716, 2245, 2933. ${ }^{1} \mathrm{H}-\mathrm{NMR}$ $\left(\mathrm{CDCl}_{3}, 400 \mathrm{MHz}\right) \delta: 1.47(\mathrm{t}, 3 \mathrm{H}, J=7.3 \mathrm{~Hz}), 1.71-1.93(\mathrm{~m}, 4 \mathrm{H}), 2.40(\mathrm{t}, 2 \mathrm{H}, J=6.8 \mathrm{~Hz}), 3.03(\mathrm{t}, 2 \mathrm{H}$, $J=7.7 \mathrm{~Hz}), 4.53(\mathrm{q}, 2 \mathrm{H}, J=7.3 \mathrm{~Hz}), 7.59(\mathrm{t}, 1 \mathrm{H}, J=6.8 \mathrm{~Hz}), 7.71(\mathrm{t}, 1 \mathrm{H}, J=7.3 \mathrm{~Hz}), 7.79(\mathrm{~d}, 1 \mathrm{H}$, $J=8.12 \mathrm{~Hz}), 8.04(\mathrm{~s}, 1 \mathrm{H}), 8.17(\mathrm{~d}, 1 \mathrm{H} \mathrm{J}=8.1 \mathrm{~Hz}) .{ }^{13} \mathrm{C}-\mathrm{NMR}\left(\mathrm{CDCl}_{3}, 100 \mathrm{MHz}\right) \delta: 14.5,17.3,25.4,30.4$, $32.1,62.4,119.7,127.2,128.5,129.0,129.9,130.0,133.3,137.5,146.2,150.0,166.9$. GC-MS (70 eV): $\mathrm{m} / \mathrm{z}$ : 282 ([M+ $], 9), 253$ (29), 228 (34), 209 (38), 209 (38), 182 (19), 170 (23), 168 (48), 154 (73), 143 (100), 142 (18), 140 (18), 115 (33), 91 (15). Anal. Calcd. for $\mathrm{C}_{17} \mathrm{H}_{18} \mathrm{~N}_{2} \mathrm{O}_{2}$ (282.34): C, 72.32; $\mathrm{H}, 6.43 ; \mathrm{N}, 9.92$. Found: $\mathrm{C}$, 72.36; H, 6.47; N, 9.89 .

Compound 4af. Pale yellow oil. IR ( $\mathrm{cm}^{-1}$, neat): 1072, 1162, 1460, 1617, 1720, 2958. 1H-NMR ( $\mathrm{CDCl}_{3}$, $400 \mathrm{MHz}) \delta: 0.89-1.03(\mathrm{~m}, 6 \mathrm{H}), 1.46(\mathrm{~d}, 3 \mathrm{H}, J=6.4 \mathrm{~Hz}), 1.66-1.79(\mathrm{~m}, 3 \mathrm{H}), 1.80-1.93(\mathrm{~m}, 1 \mathrm{H}), 2.93(\mathrm{dt}$, $2 \mathrm{H}, J=7.7,2.1 \mathrm{~Hz}), 5.19-5.31(\mathrm{~m}, 1 \mathrm{H}), 7.58(\mathrm{t}, 1 \mathrm{H}, J=8.1 \mathrm{~Hz}), 7.71(\mathrm{t}, 1 \mathrm{H}, J=8.1 \mathrm{~Hz}), 7.79(\mathrm{~d}, 1 \mathrm{H}$, $J=8.1 \mathrm{~Hz}), 8.05(\mathrm{~s}, 1 \mathrm{H}), 8.24(\mathrm{~d}, 1 \mathrm{H}, J=8.1 \mathrm{~Hz}) .{ }^{13} \mathrm{C}-\mathrm{NMR}\left(\mathrm{CDCl}_{3}, 100 \mathrm{MHz}\right) \delta: 10.2,14.3,19.8,24.8$, 29.1, 35.0, 74.8, 127.5, 128.2, 129.0, 129.8, 130.3, 133.6, 137.8, 146.0, 151.6, 167.1. GC-MS (70 eV): $\mathrm{m} / z: 271$ ([M+ $\left.\left.\mathrm{M}^{+}\right], 8\right), 215(20), 214$ (100), 200 (20), 198 (17), 182 (14), 170 (59), 168 (63), 154 (28), 143 (93), 143 (24), 115 (21), 41 (18), 29(19). Anal. Calcd. for $\mathrm{C}_{17} \mathrm{H}_{21} \mathrm{NO}_{2}$ (271.36): C, 75.25; $\mathrm{H}, 7.80 ; \mathrm{N}$, 5.16. Found: $\mathrm{C}, 75.21 ; \mathrm{H}$, 7.77; N, 5.19 .

Compound 4ba. Pale yellow oil. IR ( $\mathrm{cm}^{-1}$, neat): 1070, 1177, 1479, 1616, 1723, 2975. ${ }^{1} \mathrm{H}-\mathrm{NMR}\left(\mathrm{CDCl}_{3}\right.$, $400 \mathrm{MHz}) \delta: 1.31(\mathrm{t}, 3 \mathrm{H}, J=7.3 \mathrm{~Hz}), 1.45(\mathrm{t}, 3 \mathrm{H}, J=7.3 \mathrm{~Hz}), 2.99(\mathrm{q}, 2 \mathrm{H}, J=7.3 \mathrm{~Hz}), 4.51(\mathrm{q}, 2 \mathrm{H}$, $J=7.3 \mathrm{~Hz}), 7.57-7.63(\mathrm{~m}, 1 \mathrm{H}), 7.73-7.76(\mathrm{~m}, 1 \mathrm{H}), 7.93(\mathrm{~s}, 1 \mathrm{H}), 8.08(\mathrm{~d}, 1 \mathrm{H}, J=9.0 \mathrm{~Hz}) .{ }^{13} \mathrm{C}-\mathrm{NMR}\left(\mathrm{CDCl}_{3}\right.$, $100 \mathrm{MHz}) \delta: 14.5,15.2,25.9,62.4,125.9,129.7,130.6,131.6,134.0,135.5,136.7,144.3,150.9,166.8$. GC-MS $(70 \mathrm{eV}): m / z: 263\left(\left[\mathrm{M}^{+}\right], 26\right), 236(25), 234$ (71), 218 (15), 216 (16), 192 (30), 191 (54), 190 (100), 189 (59), 188 (57), 163 (19), 154 (52), 153 (14), 128 (14), 126 (18). Anal. Calcd. for $\mathrm{C}_{14} \mathrm{H}_{14} \mathrm{ClNO}_{2}$ (263.72): C, 63.76; $\mathrm{H}, 5.35 ; \mathrm{N}, 5.31$. Found: $\mathrm{C}, 63.80 ; \mathrm{H}, 5.38 ; \mathrm{N}, 5.27$.

Compound 4bg. Pale yellow oil. IR ( $\mathrm{cm}^{-1}$, neat): 1072, 1173, 1481, 1615, 1721, 2978. ${ }^{1} \mathrm{H}-\mathrm{NMR}\left(\mathrm{CDCl}_{3}\right.$, $400 \mathrm{MHz}) \delta: 1.46(\mathrm{t}, 3 \mathrm{H}, J=7.3 \mathrm{~Hz}), 2.64(\mathrm{~s}, 3 \mathrm{H}), 4.52(\mathrm{q}, 2 \mathrm{H}, J=7.3 \mathrm{~Hz}), 7.60(\mathrm{dd}, 1 \mathrm{H}, J=9.4,2.6 \mathrm{~Hz})$, $7.20(\mathrm{~d}, 1 \mathrm{H}, J=2.6 \mathrm{~Hz}), 7.91(\mathrm{~s}, 1 \mathrm{H}), 8.09(\mathrm{~d}, 1 \mathrm{H}, J=8.6 \mathrm{~Hz}) .{ }^{13} \mathrm{C}-\mathrm{NMR}\left(\mathrm{CDCl}_{3}, 100 \mathrm{MHz}\right) \delta: 14.5,20.0$, 62.3, 125.6, 129.6, 130.6, 131.3, 131.7, 134.2, 137.1, 144.4, 150.4, 166.6. GC-MS (70 eV): $m / z: 249$ ([M+ $\left.{ }^{+}, 9\right)$, 220 (18), 179 (31), 178 (15), 177 (100), 176 (18), 175 (18), 141 (24), 140 (40). Anal. Calcd. for $\mathrm{C}_{13} \mathrm{H}_{12} \mathrm{ClNO}_{2}$ (249.69): C, 62.53; H, 4.84; N, 5.61. Found: C, 62.50; H, 4.81; N, 5.58.

Compound 4ca. Pale brown oil. IR ( $\mathrm{cm}^{-1}$, neat): 1057, 1126, 1435, 1633, 1730, 2977. ${ }^{1} \mathrm{H}-\mathrm{NMR}\left(\mathrm{CDCl}_{3}\right.$, $400 \mathrm{MHz}) \delta: 1.36(\mathrm{t}, 3 \mathrm{H}, J=7.3 \mathrm{~Hz}), 1.47(\mathrm{t}, 3 \mathrm{H}, J=7.3 \mathrm{~Hz}), 3.04(\mathrm{q}, 2 \mathrm{H}, J=7.3 \mathrm{~Hz}), 4.54(\mathrm{q}, 2 \mathrm{H}$, $J=7.3 \mathrm{~Hz}), 7.74(\mathrm{~d}, 1 \mathrm{H}, J=8.9 \mathrm{~Hz}), 7.92(\mathrm{~d}, 1 \mathrm{H}, J=8.9 \mathrm{~Hz}), 8.11(\mathrm{~s}, 1 \mathrm{H}), 8.49(\mathrm{~s}, 1 \mathrm{H}) .{ }^{13} \mathrm{C}-\mathrm{NMR}\left(\mathrm{CDCl}_{3}\right.$, 100MHz) $\delta: ~ 14.4,15.0,25.8,29.8,32.9,62.3,121.3,123.6,123.7,126.7,127.8,127.9,128.0,128.1,128.4$, 130.4, 131.0, 131.7, 136.0, 137.7, 144.9, 152.3, 166.6. GC-MS (70 eV): $m / z: 297$ ([M+ $], 21), 268$ (82), 225 (36), 224 (100), 223 (66), 222 (96), 197 (21), 196 (14), 154 (31). Anal. Calcd. for $\mathrm{C}_{15} \mathrm{H}_{14} \mathrm{~F}_{3} \mathrm{NO}_{2}$ (297.28): C, $60.61 ; \mathrm{H}, 4.75 ; \mathrm{N}, 4.71$. Found: C, 60.57; H, 4.78; N, 4.68.

Compound 4dh. Yellow oil. IR ( $\mathrm{cm}^{-1}$, neat): 1052, 1119, 1431, 1606, 1638, 1728, 2971. ${ }^{1} \mathrm{H}-\mathrm{NMR}\left(\mathrm{CDCl}_{3}\right.$, $400 \mathrm{MHz}) \delta: 1.46(\mathrm{t}, 3 \mathrm{H}, J=7.3 \mathrm{~Hz}), 2.40-2.48(\mathrm{~m}, 2 \mathrm{H}), 2.53(\mathrm{~s}, 3 \mathrm{H}), 3.05-3.11(\mathrm{~m}, 2 \mathrm{H}), 4.52(\mathrm{q}, 2 \mathrm{H}$, $J=7.3 \mathrm{~Hz}), 4.97-5.07(\mathrm{~m}, 2 \mathrm{H}), 5.80-5.93(\mathrm{~m}, 1 \mathrm{H}), 7.50-7.55(\mathrm{~m}, 2 \mathrm{H}), 7.94(\mathrm{~s}, 1 \mathrm{H}), 8.09(\mathrm{~d}, 1 \mathrm{H}, J=9.4 \mathrm{~Hz})$. ${ }^{13} \mathrm{C}-\mathrm{NMR}\left(\mathrm{CDCl}_{3}, 100 \mathrm{MHz}\right)$ 8: 14.5, 22.0, 32.4, 35.3, 62.3, 115.9, 126.0, 129.1, 129.5, 132.2, 133.7, 137.2, 137.6, 138.6, 144.5, 149.2, 166.9. GC-MS (70 eV): m/z: 264 (7), 235 (14), 197 (37), 193 (100), 191 (50), 177 (11), 142 (11), 30 (10). Anal. Calcd. for $\mathrm{C}_{17} \mathrm{H}_{19} \mathrm{NO}_{2}$ (269.34): C, 75.81; H, 7.11; N, 5.20. Found: C, 75.77; $\mathrm{H}, 7.08 ; \mathrm{N}, 5.22$.

Compound 4di. Yellow oil. IR ( $\mathrm{cm}^{-1}$, neat): 697, 825, 1076, 1170, 1286, 1455, 1492, 1626, 1724, 2967. ${ }^{1} \mathrm{H}-\mathrm{NMR}\left(\mathrm{CDCl}_{3}, 400 \mathrm{MHz}\right) \delta: 1.25(\mathrm{t}, 3 \mathrm{H}, J=7.3 \mathrm{~Hz}), 2.53(\mathrm{~s}, 3 \mathrm{H}), 2.96(\mathrm{t}, 2 \mathrm{H}, J=7.3 \mathrm{~Hz}), 5.51(\mathrm{~s}, 2 \mathrm{H})$, 7.31-7.42 (m, 3H), 7.48-7.56 (m, 4H), $7.94(\mathrm{~s}, 1 \mathrm{H}), 8.07(\mathrm{~d}, 1 \mathrm{H}, J=8.5 \mathrm{~Hz}) .{ }^{13} \mathrm{C}-\mathrm{NMR}\left(\mathrm{CDCl}_{3}, 100 \mathrm{MHz}\right)$ $\delta: 15.4,22.0,25.9,67.8,126.0,128.6,128.8,128.9,129.3,129.6,132.1,135.8,135.9,136.0,138.5,144.5,149.2$, 166.9. GC-MS (70 eV): m/z: 210 (24), 196 (18), 168 (100), 167 (75), 165 (31), 152 (19), 141 (18), 114 (14), 90 
(73), 65 (28). Anal. Calcd. for $\mathrm{C}_{20} \mathrm{H}_{19} \mathrm{NO}_{2}$ (305.38): C, 78.66; H, 6.27; N, 4.59. Found: C, 78.69; H, 6.24; $\mathrm{N}, 4.62$.

Acknowledgments: The authors thank the University of Camerino for financial support.

Conflicts of Interest: The authors declare no conflict of interest.

\section{Abbreviations}

The following abbreviations are used in this manuscript:

TMG 1,1,3,3-Tetramethylguanidine

DBU 1,5-Diazabiciclo[5.4.0]undec-5-ene

BEMP 2-tert-Butylimino-2-diethylamino-1,3-dimethylperhydro-1,3,2-diazaphosphorine

TBD 1,5,7-Triazabicyclo[4.4.0]dec-5-ene

\section{References and Note}

1. Marella, A.; Tanwar, O.P.; Saha, R.; Ali, M.R.; Srivastava, S.; Akhter, M.; Shaquiquzzaman, M.; Alam, M.M. Quinoline: A versatile heterocyclic. Saudi Pharm. J. 2013, 21, 1-12. [CrossRef] [PubMed]

2. Afzal, O.; Kumar, S.; Haider, M.R.; Ali, M.R.; Kumar, R.; Jaggi, M.; Bawa, S. A review on anticancer potential of bioactive heterocycle quinoline. Eur. J. Med. Chem. 2015, 97, 871-910. [CrossRef] [PubMed]

3. Prajapati, S.M.; Patel, K.D.; Vekariya, R.H.; Panchal, S.N.; Patel, H.D. Recent advances in the synthesis of quinolines: A review. RSC Adv. 2014, 4, 24463-24476. [CrossRef]

4. Ciufolini, M.A.; Roschangar, F. Practical total synthesis of (+)-camptothecin: The full story. Tetrahedron 1997, 53, 11049-11060. [CrossRef]

5. Manfredini, S.; Vertuani, S.; Pavan, B.; Vitali, F.; Scaglianti, M.; Bortolotti, F.; Biondi, C.; Scatturin, A.; Prasad, P.; Dalpiaz, A. Design, synthesis and in vitro evaluation on HRPE cells of ascorbic and 6-bromoascorbic acid conjugates with neuroactive molecules. Bioorg. Med. Chem. 2004, 12, 5453-5463. [CrossRef] [PubMed]

6. Nayyar, A.; Monga, V.; Malde, A.; Coutinhob, E.; Jaina, R. Synthesis, anti-tuberculosis activity, and 3D-QSAR study of 4-(adamantan-1-yl)-2-substituted quinolines. Bioorg. Med. Chem. 2007, 15, 626-640. [CrossRef] [PubMed]

7. Horchler, C.L.; McCauley, J.P.; Hall, J.E.; Snyder, D.H.; Moore, W.C.; Hudzik, T.J.; Chapdelaine, M.J. Synthesis of novel quinoline and quinoline-2-carboxylic acid (4-morpholin-4-yl-phenyl)amides: A late-stage diversification approach to potent 5HT1B antagonists. Bioorg. Med. Chem. 2007, 15, 939-950. [CrossRef] [PubMed]

8. Contour-Galcéra, M.-O.; Sidhu, A.; Plas, P.; Roubert, P. 3-Thio-1,2,4-triazoles, novel somatostatin sst $2 /$ sst $_{5}$ agonists. Bioorg. Med. Chem. Lett. 2005, 15, 3555-3559. [CrossRef] [PubMed]

9. Saburi, H.; Tanaka, S.; Kitamura, M. Catalytic dehydrative allylation of alcohols. Angew. Chem. Int. Ed. 2005, 44, 1730-1732. [CrossRef] [PubMed]

10. Tanaka, S.; Saburi, H.; Ishibashi, Y.; Kitamura, M. CpRu ${ }^{\mathrm{II}} \mathrm{PF}_{6} /$ Quinaldic Acid-Catalyzed Chemoselective Allyl Ether Cleavage. A Simple and Practical Method for Hydroxyl Deprotection. Org. Lett. 2004, 6, 1873-1875. [CrossRef] [PubMed]

11. Tagawa, Y.; Yamashita, K.; Higuchi, Y.; Goto, Y. Improved oxidation of active methyl group of $\mathrm{N}$-heteroaromatic compounds by selenium dioxide in the presence of tert-butyl hydroperoxide. Heterocycles 2003, 60, 953-957. [CrossRef]

12. George, I.R.; Lewis, W.; Moody, C.J. Synthesis of iodopyridone. Tetrahedron 2013, 69, 8209-8215. [CrossRef]

13. Shrader, W.D.; Celebuski, J.; Kline, S.J.; Johnson, D. Synthesis of a novel hexadentate chelating agent based on8-hydroxyquinoline. Tetrahedron Lett. 1988, 29, 1351-1354. [CrossRef]

14. Huang, H.; Jiang, H.; Chen, K.; Liu, H. A Simple and convenient copper-catalyzed tandem synthesis of quinoline-2-carboxylates at room temperature. J. Org. Chem. 2009, 74, 5476-5480. [CrossRef] [PubMed]

15. Rohlmann, R.; Stopka, T.; Richter, H.; Mancheño, O.G. Iron-catalyzed oxidative tandem reactions with TEMPO oxoammonium salts: Synthesis of dihydroquinazolines and quinolines. J. Org. Chem. 2013, 78, 6050-6064. [CrossRef] [PubMed] 
16. Bass, J.Y.; Caravella, J.A.; Chen, L.; Creech, K.L.; Deaton, D.N.; Madauss, K.P.; Marr, H.B.; McFadyen, R.B.; Miller, A.B.; Mills, W.Y.; et al. Conformationally constrained farnesoid X receptor (FXR) agonists: Heteroaryl replacements of the naphthalene. Bioorg. Med. Chem. Lett. 2011, 21, 1206-1213. [CrossRef] [PubMed]

17. Ciufolini, M.A.; Mitchell, J.W.; Rosehangar, F. Facile palladium-mediated substitution of chlorine in 2-chloroquinolines. Tetrahedron Lett. 1996, 37, 8281-8284. [CrossRef]

18. Itoh, S.; Fukui, Y.; Haranou, S.; Ogino, M.; Komatau, M.; Ohshiro, Y. Synthesis and characterization of dimethyl 9,10-dihydro-9,10-dioxobenzo[f]quinoline-2,4-dicarboxylate. Effect of the pyrrole nucleus on the reactivity of coenzyme PQQ. J. Org. Chem. 1992, 57, 4452-4457. [CrossRef]

19. Ballini, R.; Palmieri, A.; Talaq, M.A.; Gabrielli, S. Preparation of 2H-1,4-benzoxazin-2-one derivatives under heterogeneous conditions via domino process. Adv. Synth. Catal. 2009, 351, 2611-2614. [CrossRef]

20. Ballini, R.; Gabrielli, S.; Palmieri, A. $\beta$-Nitroacrylates as key starting materials for the uncatalysed one-pot synthesis of polyfunctionalized dihydroquinoxalinone derivatives, via an anti-Michael reaction. Synlett 2009, 2009, 965-967. [CrossRef]

21. Ballini, R.; Bosica, G.; Gabrielli, S.; Palmieri, A. $\beta$-Nitroacrylates and silyl enol ethers as key starting materials for the synthesis of polyfunctionalized $\beta$-nitro esters and 1,2-oxazine-2-oxides. Tetrahedron 2009, 65, 2916-2920. [CrossRef]

22. Ballini, R.; Gabrielli, S.; Palmieri, A. $\beta$-Nitroacrylates as precursors of tetrasubstituted furans in a one-pot process and under acidic solvent-free conditions. Synlett 2010, 2010, 2468-2470. [CrossRef]

23. Palmieri, A.; Gabrielli, S.; Lanari, D.; Vaccaro, L.; Ballini, R. A new one-pot synthesis of polysubstituted indoles from pyrroles and $\beta$-nitroacrylates. Adv. Synth. Catal. 2011, 353, 1425-1428. [CrossRef]

24. Palmieri, A.; Gabrielli, S.; Cimarelli, C.; Ballini, R. Fast, mild, eco-friendly synthesis of polyfunctionalized pyrroles from $\beta$-nitroacrylates and $\beta$-enaminones. Green Chem. 2011, 13, 3333-3336. [CrossRef]

25. Gabrielli, S.; Ballini, R.; Palmieri, A. $\beta$-Nitroacrylates as key building blocks for the synthesis of alkyl 3-substituted 5-oxopiperazine-2-carboxylates under fully heterogeneous conditions. Mon. Chem. 2013, 144, 509-514. [CrossRef]

26. Palmieri, A.; Gabrielli, S.; Maggi, R.; Ballini, R. $\beta$-Nitroacrylates as useful building blocks for the synthesis of alkyl indole-2-carboxylates. Synlett 2014, 25, 128-132. [CrossRef]

27. Palmieri, A.; Gabrielli, S.; Parlapiano, M.; Ballini, R. One-pot synthesis of alkyl pyrrole-2-carboxylates starting from $\beta$-nitroacrylates and primary amines. RSC Adv. 2015, 5, 4210-4213. [CrossRef]

28. Castaing, M.; Wason, S.L.; Estepa, B.; Hooper, J.F.; Willis, M.C. 2-Aminobenzaldehydes as versatile substrates for rhodium-catalyzed alkyne hydroacylation: Application to dihydroquinoline synthesis. Angew. Chem. Int. Ed. 2013, 52, 13280-13283. [CrossRef] [PubMed]

29. Haddadin, M.J.; Bou Zerdan, R.M.; Kurth, M.J.; Fettinger, J.C. Efficient syntheses of the unknown quinolino[2,3-c]cinnolines; synthesis of neocryptolepines. Org. Lett. 2010, 12, 5502-5505. [CrossRef] [PubMed]

30. Ballini, R.; Fiorini, D.; Palmieri, A. Nitroalkanes and ethyl glyoxalate as common precursors for the preparation of both $\beta$-keto esters and $\alpha, \beta$-unsaturated esters. Tetrahedron Lett. 2004, 45, 7027-7029. [CrossRef]

31. Palmieri, A.; Gabrielli, S.; Ballini, R. Low impact synthesis of $\beta$-nitroacrylates under fully heterogeneous conditions. Green Chem. 2013, 15, 2344-2348. [CrossRef]

32. The BEMP on polymer support was purchased from Sigma-Aldrich (code 536490) and used directly without any manipulation.

Sample Availability: Samples of the compounds 4 are available from the authors. 